\title{
TEMA 12-2016: Cetoacidosis diabética y estado hiper- glicémico hiperosmolar: un enfoque práctico
}

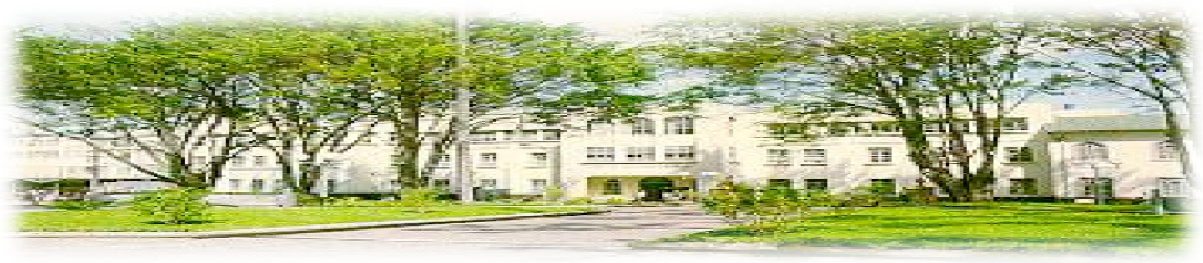

ISSN

Hospital San Juan de Dios, San José, Costa Rica. Fundado en 1845

Recibido:

$28 / 12 / 2015$

Aceptado:

$05 / 01 / 2016$

Gloriana Arroyo Sánchez ${ }^{1}$ Saúl Quirós Cárdenas ${ }^{2}$

${ }^{1}$ Médico General. Universidad de Costa Rica. Correo electrónico: gloriana.arroyo@gmail.com

${ }^{2}$ Médico Residente de Medicina Interna. Programa de Estudios de Postgrado UCR-CENDEISSS.

Hospital San Juan de Dios. San José, Costa Rica

\section{RESUMEN}

La cetoacidosis diabética (CAD) y elestado hiperglicémicohiperosmolar (EHH), en conjunto conocidas como crisis o emergencias hiperglicémicas, tradicionalmente han sido asociadas a diabetes mellitus mal controlada. Ambas son entidades diferentes desde el punto de vista del cuadro clínico y los hallazgos de laboratorio, no obstante, en ocasiones pueden sufrir un traslape entre ellas. Producto de lo antes dicho,es importante que el personal médico tenga una adecuada comprensión de la fisiopatología, los factores desencadenantes, las manifestaciones clínicas y de laboratorio de dichas condiciones, de manera que se pueda realizar un diagnóstico correcto que conduzca a la instauración de tratamiento temprano y adecuado.

\section{PALABRAS CLAVE}

Cetoacidosis, hiperglicemia, hiperosmolaridad, diabetes mellitus, emergencias, crisis.

\section{ABSTRACT}

The diabetic ketoacidosis (DKA) and the hyperglycemic hyperosmolar state (HHS), known as hyperglycemic crisis or emergencies, have traditionally been associated with poorly controlled diabetes mellitus. Both are different entities with particular clinical and laboratory findings, which sometimes may overlap. Hence the importance of the medical professionals to have an adequate understanding of the pathophysiology, triggers, clinical and laboratory manifestations of these conditions, so that they can make a correct diagnosis leading to the establishment of early and appropriate treatment. 


\section{KEY WORDS}

Ketoacidosis, hyperglycemia, hyperosmolar, diabetes mellitus, emergencies, crisis.

\section{INTRODUCCIÓN}

La cetoacidosis diabética y el estado hiperglicémico hiperosmolar constituyen las emergencias hiperglicémicas más temidas y potencialmente fatales en los pacientes diabéticos con enfermedad mal controlada. Ambas complicaciones metabólicas agudas y severas, pueden llevar a morbilidad neurológica significativa y muerte, con peor pronóstico cuando se presentan de forma simultánea en aproximadamente un tercio de los $\operatorname{casos}^{(1,2,3,4)}$.

En el contexto actual de un dramático aumento de la prevalencia general de diabetes alrededor del mundo, resulta fundamental la comprensión de estas entidades, que favorezca un diagnóstico oportuno con la debida instauración del tratamiento requerido ${ }^{(5)}$.

La presente revisión ofrece una descripción de los principales aspectos relacionados con dichas patologías, de manera que facilite un enfoque práctico y manejo adecuado de los pacientes con este diagnóstico.

\section{DISCUSIÓN}

\section{DEFINICIONES}

La cetoacidosis diabética (CAD) y estado hiperglicémico hiperosmolar (EHH) representan complicaciones severas de la diabetes mellitus. Constituyen estados hipeglicémicos que se diferencian clínicamente por la severidad de la deshidratación, y si la cetosis y acidosis metabólica están o no presentes: la cetoacidosis se caracteriza por cetonemia y acidosis con brecha aniónica elevada, mientras que el estado hiperglicémicohiperosmolar como su nombre lo indica por hiperosmolaridad sérica y deshidratación severa ${ }^{(2,3)}$.

Estas dos entidades se producen en el contexto de deficiencia relativa o absoluta de insulina asociado a niveles excesivos de hormonas contrarreguladoras, depleción progresiva de volumen, así como pérdida de electrolitos ${ }^{(5)}$.

\section{EPIDEMIOLOGÍA}

La prevalencia y carga financiera de la diabetes son enormes y van en aumento. Según datos del Centro de Control de Enfermedades (CDC, por sus siglas en inglés) para el año 2014, $9.3 \%$ de la población estadounidense padecía de diabetes. Por su parte la realidad en Costa Rica no dista de lo anteriormente descrito, con una incidencia calculada para el año 2009 de 5-6\% de la población mayor de 20 años, constituyendo la patología que mayor costo genera en la atención hospitalaria y la segunda causa de consulta externa en el adulto $^{(1,6,7)}$.

Las emergencias hiperglicémicas asociadas a la diabetes forman parte de este espectro. La cetoacidosis diabética tiene nuna incidencia estimada en Estados Unidos de 4-8 episodios por cada 1000 ingresos de pacientes por diabetes, la misma es mayor entre niños jóvenes y personas con bajo nivel socioeconómico. Mientras, el estado hiperglicémico hiperosmolar se calcula que se presenta en $1 \%$ de los ingresos por diabetes ${ }^{(1,3)}$.

La cetoacidosis diabética es más común en individuos jóvenes, mientras el estado hiperglicémico hiperosmolar se considera ocurre más frecuentemente en personas mayores. Sin embargo esto no significa que sus presentaciones se limitan a estos grupos de edad, en tanto se ha descrito que al menos un $20 \%$ de los pacientes con EHH son menores de 30 años, e incluso se ha visto en niños de 18 meses de edad ${ }^{(5)}$.

Según la literatura, la mortalidad por el EHH es mucho mayor (10-20\%) con respecto a la asociada a CAD (1-5\%), en el caso de la segunda, esta constituye la causa de muerte más frecuente entre niños y adolescentes que padecen de diabetes tipo 1 , secundaria específicamente al edema cerebral en la mayoría de los casos. Se considera que en los casos de EHH la muerte es debida a una causa subyacente en lugar de la complicación de la condición en sí misma, por lo cual siempre debe buscarse exhaustivamente la causa precipitante. La combinación de ambas patologías conlleva un peor pronóstico a pesar del hecho de que estos pacientes tienden a ser más jóvenes ${ }^{(1,4,5)}$.

\section{FISIOPATOLOGÍA}

El desarrollo de estas emergencias hiperglicémicas resulta de alteraciones en el metabolismo de la glucosa y balance de fluidos con o sin cetogénesis asociada, dependiendo de la entidad en particular. La hiperglicemia provoca pérdidas 
urinarias de agua y electrolitos (sodio, potasio, cloruro) llevando a una depleción del volumen extracelular,con la aparición consecuente de deshidratación $^{(2,8)}$

La cetoacidosis diabética se caracteriza por una tríada de trastornos metabólicos: hiperglicemia no controlada, acidosis metabólica de moderada a severa y aumento en la concentración total de cetonas. Entre los factores que favorecen dichas alteraciones se pueden mencionar: deficiencia de insulina (total o relativa) que lleva a la hiperglicemia y lipólisis descontrolada con cetogénesis subsecuente, niveles elevados de hormonas contrarreguladoras, deshidratación progresiva $\mathrm{y}$ pérdida de electrolitos secundario a glucosuria persistente y vómitos, con una disminución final de la tasa de filtración glomerular debido a que la diuresis osmótica sostenida lleva a hipovolemia ${ }^{(5}$,

Por su parte el estado hiperglicémico hiperosmolar está caracterizado por elevaciones extremas de la glicemia e hiperosmolaridad sérica en un contexto de mayor deshidratación (pérdida de un $10-15 \%$ del peso corporal total o 7-12L) sin cetosis significativa, alteraciones metabólicas que así como la CAD están asociadas a los factores sinérgicos anteriormente descritos, con algunas diferencias: en el EHH se han descrito mayores concentraciones de insulina con menores niveles de glucagón (aumento en la proporción insulina/glucagón), es decir hay suficiente producción pancreática de insulina para prevenir la lipólisis requerida para generar cetosis y acidemia, pero no suficiente para prevenir la hiperglicemia $^{(1,2,5,9,10)}$.

Si bien tradicionalmente ambas condiciones se entendían como entidades separadas, actualmente se acepta la existencia de un traslape entre ambos síndromes, denominado cetoacidosis hiperosmolar con mortalidad mayor que la descrita en ambas entidades por aparte ${ }^{(5,10)}$.

\section{FACTORES DESENCADENANTES}

Los principales desencadenantes de CAD y EHH son la falta de insulina exógena (por mala adherencia al tratamiento o dosis subterapéuticas) e infección (del tracto urinario, neumonía, absceso dental, en piel, sepsis, síndromes virales, enfermedad pélvica inflamatoria, otitis externa maligna, entre otras). Se han visto también asociados a condiciones médicas subyacentes que provocan liberación de hormonas contrarreguladores tales como apendicitis, pancreatitis, inflamación abdominal, trauma, embarazo, enfermedad cerebrovascular o infarto al miocardio. Así como a la utilización de algunos fármacos y sustancias causantes de descompensaciones metabólicas tales como: cocaína, alcohol, fármacos simpaticomiméticos, antipsicóticos atípicos, corticoesteroides y diuréticos tiazídicos, entre otros ${ }^{(1,3,9)}$.

Hasta un $15 \%$ de los casos de CAD se tratan de pacientes no conocidos diabéticos sin ninguna condición subyacente, quienes debutan con la enfermedad de esta manera, específicamente en niños y jóvenes diabéticos tipo $1^{(10)}$.

\section{MANIFESTACIONES CLÍNICAS}

Los pacientes con CAD pueden presentar síntomas generales de hiperglicemia (poliuria, polidipsia, polifagia, pérdida de peso) en los días previos al establecimiento propiamente del síndrome. Los síntomas propios de la cetoacidosis se presentan en menos de 24 horas y comprenden: respiración de Kussmaul, aliento a cetonas, contracción del volumen del fluido extracelular, náuseas, vómitos y dolor abdominal, alteraciones en el nivel de consciencia (que varía según la severidad). ${ }^{(8,10)}$

En el caso del EHH, su presentación es más larvada, ocurriendo de días a semanas. Los pacientes presentan una contracción del volumen del fluido extracelular más profunda, con una alteración severa del nivel de consciencia (proporcional a la elevación de la osmolaridad plasmática). Pueden adicionalmente tener una variedad de presentaciones neurológicas incluyendo convulsiones o un estado similar al de pacientes con eventos cerebrovasculares, que resuelven una vez que la osmolaridad retorna a los niveles normales ${ }^{(8,10,11)}$

Conviene recordar que en ambas condiciones, también puede haber evidencia clínica de una condición precipitante ${ }^{(8)}$.

\section{DIAGNÓSTICO Y ABORDAJE INICIAL}

Los criterios diagnósticos para CAD y EHH (Tabla I) se basan en diferentes valores de laboratorio así como algunas manifestaciones clínicas características $^{(1,3,12)}$

De tal manera que la valoración inicial de estos pacientes debe incluir la toma glucosa plasmática, $\mathrm{pH}$ venoso/arterial (en pacientes más graves se recomienda la determinación por medio de gases 
arteriales, mientras que en el resto de pacientes una muestra de gases venosos es adecuada en tanto el $\mathrm{pH}$ venoso es típicamente 0.015-0.03 menor que el arterial), bicarbonato sérico, cetonas en orina y sangre, osmolaridad sérica efectiva, brecha aniónica, balance de fluidos, así como la determinación del estado mental y complicaciones o factores precipitantes asociados ${ }^{(8,12)}$

Tabla

Criterios diagnósticos para CAD y EHH

\begin{tabular}{|c|c|c|c|c|}
\hline & \multicolumn{3}{|c|}{ CAD } & \multirow[t]{2}{*}{ ЕНН } \\
\hline & Leve & $\begin{array}{l}\text { Mode- } \\
\text { rada }\end{array}$ & Severa & \\
\hline $\begin{array}{l}\text { Glucosa } \\
\text { plasmá- } \\
\text { tica } \\
(\mathrm{mg} / \mathrm{dL})\end{array}$ & $>250$ & $>250$ & $>250$ & $>600$ \\
\hline $\mathrm{pH}$ & $\begin{array}{c}7.25- \\
7.3\end{array}$ & $\begin{array}{l}7.0- \\
7.24\end{array}$ & $<7.0$ & $>7.3$ \\
\hline $\begin{array}{l}\text { Bicarbo- } \\
\text { nato } \\
\text { sérico } \\
(\mathrm{mEq} / \mathrm{L})\end{array}$ & $15-18$ & $10-15$ & $<10$ & $>18$ \\
\hline $\begin{array}{c}\text { Cetonas } \\
\text { (séricas } \\
\text { o urina- } \\
\text { rias) }\end{array}$ & $(+)$ & $(+)$ & $(+)$ & $\begin{array}{l}\text { Míni- } \\
\text { ma o } \\
(-)\end{array}$ \\
\hline $\begin{array}{c}\text { Brecha } \\
\text { aniónica }\end{array}$ & $>10$ & $>12$ & $>12$ & $\begin{array}{c}\text { Varia- } \\
\text { ble }\end{array}$ \\
\hline $\begin{array}{l}\text { Osmola- } \\
\text { ridad }\end{array}$ & $\begin{array}{c}\text { Varia- } \\
\text { ble }\end{array}$ & $\begin{array}{c}\text { Varia- } \\
\text { ble }\end{array}$ & $\begin{array}{l}\text { Varia- } \\
\text { ble }\end{array}$ & $>320$ \\
\hline $\begin{array}{l}\text { Estado } \\
\text { mental }\end{array}$ & Alerta & $\begin{array}{c}\text { Alerta } \\
\text { Somno- } \\
\text { liento }\end{array}$ & $\begin{array}{c}\text { Estupor } \\
\text { coma }\end{array}$ & $\begin{array}{l}\text { Estu- } \\
\text { por } \\
\text { coma }\end{array}$ \\
\hline $\begin{array}{l}\text { Des- } \\
\text { hidrata- } \\
\text { ción }\end{array}$ & $6 \mathrm{~L}$ & & & $9 \mathrm{~L}$ \\
\hline
\end{tabular}

Fuente: EmergMedClin N Am 32 (2014) 437-452

Hormones 2011, 10(4):250-260

Se debe tener en consideración que la evaluación del paciente basada en los criterios antes mencionados facilita el diagnóstico, pero puede ocurrir que en condiciones específicas no se cumplan a cabalidad, tal y como se observa en el fenómeno de "cetoacidosis diabética euglicémica", una entidad muy poco frecuente ( $10 \%$ de los casos de CAD), descrita por primera vez en 1973 y definida como niveles de glucosa $<250 \mathrm{mg} / \mathrm{dL}$ en el contexto de una cetoacidosis diabética ${ }^{(11)}$.

\section{DIAGNÓSTICO DIFERENCIAL}

Si bien la presentación de los estados hiperglicémicos en la mayoría de los casos se asocia a pacientes diabéticos con mal control, resulta necesario por medio de historia clínica y hallazgos al examen físico y laboratorio, descartar otros posibles diagnósticos que pueden llevar a alteraciones metabólicas similares.

La cetoacidosis sola no indica diagnóstico exclusivo de $\mathrm{CAD}$. Se debe hacer diagnóstico diferencial con cetosis por ayuno prolongado, y cetoacidosis alcohólica. De igual manera al enfrentarse a un paciente con acidosis metabólica con brecha aniónica elevada, se tienen que considerar otras causas alternativas entre ellas acidosis láctica secundaria a ejercicio extenuante, cáncer, sepsis, fallo respiratorio, entre otras. Es importante destacar que en caso de sospechar CAD, se debe descartar como diagnóstico un EHH, pues alrededor de un $50 \%$ de las veces estos se presentan además con cetosis $^{(8,12)}$.

\section{TRATAMIENTO}

El tratamiento de ambas entidades se basa en la corrección de las alteraciones metabólicas subyacentes: hiperglicemia, producción de cetoácidos, trastornos electrolíticos; de la depleción de volumen, así como del manejo de la causas subyacente $o$ factores desencadenantes ${ }^{(1,3)}$.

\section{Resucitación con fluidos}

Se debe iniciar inmediatamente posterior al diagnóstico. Para la resucitación inicial se recomienda la utilización de solución salina $0.9 \%$. En pacientes con datos claros de shock o falla cardíaca se debe administrar $1 \mathrm{~L}$ de solución salina en los primeros 30-60 minutos, y pasar 15$20 \mathrm{~mL} / \mathrm{Kg} /$ hora $(\mathrm{h})$ durante las primeras 2 horas. En las siguientes 2-6 horas administrar otros 2L y finalmente $2 \mathrm{~L}$ adicionales durante las siguientes 6-12 horas. Esta estrategia inicial repleta aproximadamente $50 \%$ de las pérdidas de líquido en las primeras 12 horas, mientras que el porcentaje restante de requerimientos se repone en las 12-36 horas siguientes a la admisión ${ }^{(1)}$.

Posterior a esto, la solución utilizada para la subsecuente reposición debe ser seleccionada de manera individualizada tomando en consideración aspectos como el grado de hidratación, electrolitos séricos y gasto urinario ${ }^{(1,6)}$.

Tanto en CAD como en el EHH, cuando los niveles plasmáticos de glucosa caen entre 250 -
Rev Cl EMed UCR

www.revistaclinicahsjd.ucr.ac.cr 
$300 \mathrm{mg} / \mathrm{dL}$, se deben iniciar fluidos que contengan dextrosa, para evitar hipoglicemia y permitir la continuación de la terapia con insulina. Se recomienda empezar con dextrosa al $5 \%$ y si los niveles de glucosa continúan descendiendo aumentar la concentración al 10\% $\%^{(1,2,6)}$

Tratar pacientes con compromiso cardíaco o renal es un reto, debido a que se tiene que establecer un fino balance entre el déficit y sobrecarga de volumen. En estos casos se requiere un monitoreo estricto ${ }^{(2)}$.

\section{Insulina}

No se debe iniciar al momento del diagnóstico, sino que se recomienda primero administrar la terapia con fluidos, esperar a que los resultados de electrolitos estén disponibles (para tener seguridad de que el potasio sérico se encuentra al menos por encima de $3.3 \mathrm{mEq} / \mathrm{L}$ ) y hasta ese momento empezar el tratamiento con insulina ${ }^{(1,6)}$

El esquema de elección es la administración de insulina regular, iniciando con un bolo de 0.1 $\mathrm{U} / \mathrm{Kg}$ seguido por una tasa de infusión de 0.1 $\mathrm{U} / \mathrm{Kg} / \mathrm{h}$, o en su defecto omitir el bolo e iniciar infusión continua a $0.14 \mathrm{U} / \mathrm{Kg} / \mathrm{h}$ intravenosa (IV), sin que se haya logrado mostrar diferencia en eficacia entre estas dos modalidades. Con lo cual se espera una disminución de la glucosa sérica en $50-70 \mathrm{mg} / \mathrm{dL} / \mathrm{h}^{(1,6)}$.

Si no se logra el descenso de la glicemia de la forma esperada, se sospecha resistencia a la insulina secundaria, por lo cual se sugiere duplicar la infusión de insulina cada hora (iniciando a $0.2 \mathrm{U} / \mathrm{Kg} / \mathrm{h}$ ) hasta que se alcance un descenso estable de los valores de glucosa sérica. De igual manera, cuando se inician las soluciones con dextrosa la infusión de insulina se puede reducir a la mitad $(0.02-0.05 \mathrm{U} / \mathrm{Kg} / \mathrm{h})$ con la meta de mantener la glicemia entre $150-200 \mathrm{mg} / \mathrm{dL}$ hasta la corrección de la brecha aniónica ${ }^{(1)}$.

En pacientes ya capaces de tolerar la vía oral y en los cuales se desea suspender la infusión de insulina, se deben cumplir algunos criterios antes de poder hacerlo: en CAD tener glicemia $<200$ $250 \mathrm{mg} / \mathrm{dL}$ con brecha aniónica normal y $\mathrm{pH}$ $>7.30$ y bicarbonato $\geq 18 \mathrm{mEq} / \mathrm{L}$; mientras que en EHH una glicemia entre 250-300 mg/dL. En los pacientes ya conocidos diabéticos se recomienda continuar con sus regímenes previos, mientras que en debutantes se puede iniciar un esquema de insulina multidosis subcutánea (0.5-0.8 $\mathrm{U} / \mathrm{Kg} / \mathrm{d})^{(1)}$

\section{Potasio}

La reposición de potasio en el contexto de CAD o EHH siempre es necesaria, lo que varía es su momento de inicio. Es guiada por la determinación de los electrolitos iniciales y la presencia o no de una diuresis adecuada. La meta es mantener los niveles de potasio entre $4-5 \mathrm{mEq} / \mathrm{L} \mathrm{du}$ rante la terapia ${ }^{(1,3,6)}$.

De acuerdo al valor inicial de potasio, si está $>5.3 \mathrm{mEq} / \mathrm{L}$ no se recomienda reposición, y tomar control en $1 \mathrm{~h}$, si se encuentra entre 4.0-5.3 $\mathrm{mEq} / \mathrm{L}$ se debe agregar a los fluidos IV Cloruro de Potacio $(\mathrm{KCl}) 10 \mathrm{mEq} / \mathrm{L} / \mathrm{h}$, con potasio entre $3.5-<4.0 \mathrm{mEq} / \mathrm{L}$ añadir $\mathrm{KCl} 20 \mathrm{mEq} / \mathrm{L} / \mathrm{h}$ a los fluidos IV, y si el potasio se encuentra $<3.5$ $\mathrm{mEq} / \mathrm{L}$ se recomienda detener la insulina y agregar $\mathrm{KCl} 20-60 \mathrm{mEq} / \mathrm{L} / \mathrm{h}$ a los fluidos $\mathrm{IV}^{(1)}$.

Mientras que de acuerdo a la función renal, en caso de falla renal u oliguria, la reposición de potasio debe reducirse y sólo debe ocurrir cuando el potasio sérico está $<4 \mathrm{mEq} / \mathrm{L}$ o con un electrocardiograma (EKG)que muestra signos de hipokalemia $^{(1,3)}$.

\section{CONCLUSIONES}

El acelerado aumento en los últimos años de la prevalencia de la diabetes mellitus alrededor del mundo, supone la necesidad de una adecuada comprensión del espectro de manifestaciones que esta patología puede tener. La cetoacidosis diabética y el estado hiperglicémicohiperosmolar constituyen parte de esta realidad, entidades que con un abordaje y manejo adecuado, pueden resolver evitando la morbimortalidad asociada. El conocimiento de la fisiopatología, las manifestaciones clínicas y de laboratorio, así como factores desencadenantes y diagnósticos diferenciales a considerar cuando se está frente a un paciente con una crisis hiperglicémica, facilita la realización de un adecuado diagnóstico y por consiguiente la instauración temprana de las medidas terapéuticas que faciliten la corrección de las alteraciones metabólicas, el estado de hidratación y causa subyacente. 


\section{BIBLIOGRAFÍA}

1. Corwell B. et al. Current Diagnosis and Treatment of Hyperglycemic Emergencies. EmergMedClin N Am 32 (2014) $437-452$

2. Maletkovic J \&Drexler A. Diabetic Ketoacidosis and Hyperglycemic Hyperosmolar State. EndocrinolMetabClin N Am 42 (2013) 677-695

3. Chaithongdi N. et al. Diagnosis and management of hyperglycemic emergencies. Hormones 2011, 10(4):250-260

4. Nyenwe E. \&Kitabchi A. Evidencebased management of hyperglycemic emergencies in diabetes mellitus. Diabetes Research and ClinicalPractice 94 (2011) 340-351

5. Steenkamp D. Adult Hyperglycemic Crisis: A Review and Perspective. CurrDiabRep (2013) 13:130-137

6. Centros para el Control y la Prevención de Enfermedades. Informe Nacional de Estadísticas de la Diabetes (National Diabetes StatisticsReport): Estimaciones sobre la diabetes y su carga en los Estados Unidos, 2014. Atlanta, GA: Departamento de Salud y Servicios Humanos de los EE.UU

7. Cedeño M. et al. Análisis epidemiológico de la Diabetes Mellitus. Revista Médica de Costa Rica y Centroamérica LXVII (509) 331-336 2009

8. Canadian Diabetes Association Clinical Practice Guidelines Expert Committee. HyperglycemicEmergencies in Adults. Can J Diabetes 37 (2013) S72eS76

9. Pasquel F \&Umpierrez G. Hyperosmolar Hyperglycemic State: A Historic Review of the Clinical Presentation, Diagnosis, and Treatment. Diabetes Care 2014; 37: 3124-3131

10. Gabriel E \&Soni S. Diabetic Ketoacidosis. HospMedClin 3 (2014) 556-566
11. Van Ness-Otunnu R \&Hack, J. Hyperglycemic crisis. TheJournal of Emergency Medicine, Vol. 45, No. 5, pp. 797-805, 2013

12. McDonald $\mathrm{C}$ et al. Differentiating between DKA and HHS. J EmergNurs 2015;41:201-7. $\square$ 0099-1767

\section{CONFLICTOS DE INTERES}

Los autores declaran que no existe ningún conflicto de interés. 